\title{
Indoor Localization based on Bluetooth Technology: A Brief Review
}

\author{
Devanshi \\ ECE Deptt \\ UIET,Panjab University \\ Chandigarh,160014,India
}

\author{
Sunil Agrawal \\ ECE Deptt \\ UIET,Panjab University \\ Chandigarh,160014,India
}

\author{
Sarvjit Singh \\ ECE Deptt \\ UIET,Panjab University \\ Chandigarh,160014,India
}

\begin{abstract}
GPS (Global Positioning System) is a widely used and a very popular system for tracking devices and finding out the location of the devices in the outdoor locations. But it is not successful for indoor localization because of reflections of the signals due to walls and presence of furniture and various other factors causing signal attenuation inside a building. Hence, WLAN, Bluetooth, RFID, Wi-Fi are being used for finding positions of devices being carried by people inside a building. Bluetooth, having low power consumption and low cost can be easily found in every single device from a cheap cell phone to a costly smart phone and laptops. In this paper, we are reviewing the recent development that has taken place in the field of Bluetooth indoor positioning and how different approaches and algorithms bring improvement in positioning accuracy and minimize the error.
\end{abstract}

\section{Keywords}

Bluetooth(BT), Golden Receiver Power Range(GRPR), Indoor localization, Positioning accuracy, Radio Map (RM), Received(Rx) power level, Received Signal Strength(RSS)

\section{INTRODUCTION}

With the advancement of technology, the demand for localization systems has been increasing tremendously. Indoor localization refers to finding the location or position of any mobile device, node or any object having a wireless link to some known points[1].Locations such as hospitals, airports, shopping malls, national libraries sometimes become confusing and complex to travel and users travelling are benefited by guidance provided by indoor localization[2].Extreme environments such as fire scenes, underground mines are installed with navigation systems to increase the chance of survival[3].Also, intelligent environments can be created by having knowledge of people's presence, number of people, movement and their behavior[4]. GPS uses satellites to find out the position and is very accurate. But this system is successful only for outdoor locations. This is because the radio signals are blocked by the walls, skyscrapers, ceilings and furniture, etc present inside the buildings. Thus, systems such as WLAN, Wi-Fi, RFID, Bluetooth are employed for indoor positioning. Out of these, Bluetooth being cheaper and easily available is used widely for positioning purposes.

Bluetooth is a wireless technology that operates in unlicensed ISM band of $2.45 \mathrm{GHz}$.It is a low cost and a low power technology that can provide both synchronous and asynchronous connection with range of about $10 \mathrm{~m}$ to $100 \mathrm{~m}$.Frequency Hopping Spread Spectrum technique is implemented to minimize effects due to signal interference.
Bluetooth devices are manufactured in 3 power classes-Class 3 transmitting at $1 \mathrm{~mW}$ (range $1 \mathrm{~m})$, Class 2 transmitting at $2.5 \mathrm{~mW}$ (range $10 \mathrm{~m}$ ) and Class 1 transmitting at $100 \mathrm{~mW}$ (range $100 \mathrm{~m}$ ).Being cheap and due to its presence in every single device from mobile phones to laptops, it is becoming popular for positioning purposes in indoor locations.

In Bluetooth, device discovery is carried out in which an inquiry is carried out by a Bluetooth node to look for the devices in its proximity[5].The device initiating inquiry is said to be inquiring device and the one configured to be discovered is scanning device. The inquiring device transmits inquiry packets by frequency hopping through slots and the scanning device responds with a Frequency Hopping Synchronization(FHS) packets after obtaining an inquiry packet. This FHS packet contains address of the device and the signal strength. This inquiry process can take up to 10.24 seconds during which no communication can take place.

\section{SIGNAL PARAMETERS IN BLUETOOTH SPECIFICATION \\ 2.1. RSSI-Received Signal Strength Indicator}

It is an 8-bit integer value that tells whether received power is within the Golden Receiver Power Range(GRPR) or not.GRPR represents power range with lower threshold$56 \mathrm{dBm}$ and upper threshold around $-40 \mathrm{dBm}$. The received power level(Rx-power level) is considered above GRPR when RSSI value is positive and negative values imply Rx-power level below GRPR and Rx-power level lies within golden range when RSSI has zero value. Fig.1 represents GRPR.

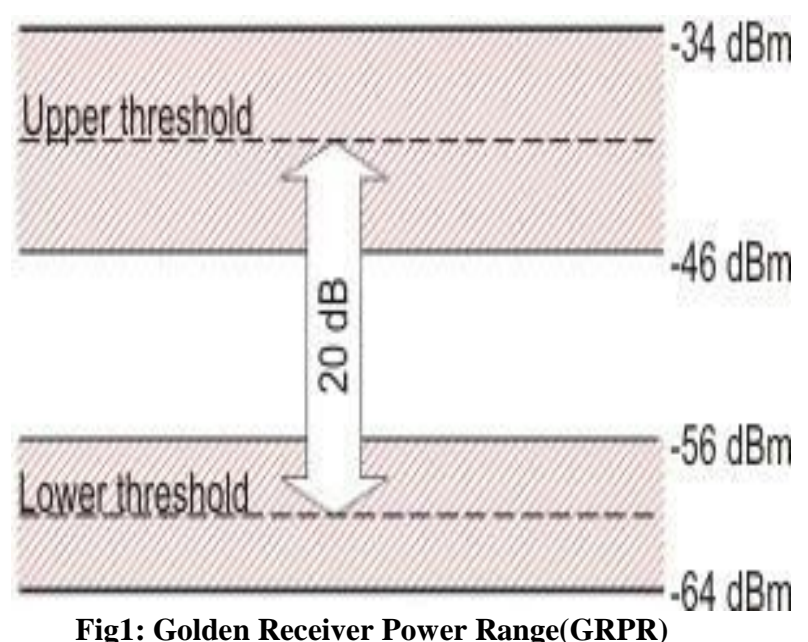




\subsection{Received (Rx) Power Level}

It is a parameter obtained indirectly from connection based RSSI value. It can be obtained if lower and upper threshold values of GRPR are already known.

$$
\begin{gathered}
R X=R S S I+G R P R_{\text {upper }} \quad \text { if } R S S I>0 \\
R X=R S S I-G R P R_{\text {lower }} \quad \text { if } R S S I<0 \\
G R P R_{\text {lower }}<R X<G R P R_{\text {upper }} \text { if } R S S I=0
\end{gathered}
$$

\subsection{Link Quality (LQ)}

It corresponds to average Bit Error Rate (BER) at receiver side. It is an 8-bit integer value showing status of a link in terms of received packets. LQ ranges between 0 and 255 .

\subsection{Transmit Power Level(TPL)}

It specifies the transmission power level of a device. The numerical values of this parameter range between 20 and $20 \mathrm{dBm}$ depending upon Bluetooth class specification.

\subsection{Inquiry based RSS}

The radio layer of Bluetooth specifications monitors Rxpower level of the inquiry response and gives corresponding RSS level. The inquiry procedure takes about 10.24 seconds. Out of the above mentioned parameters, the inquiry based RSS correlates nicely with distance calculated. This relationship is mathematically expressed as radio propagation model.

$$
d=10^{(A-R S S) / 10 * n}
$$

Here, $d$ is distance between mobile node and inquiring node. $A$ and $n$ are radio propagation constants. The $n$ can vary from 1 to 4 for indoor environment and $A$ is average inquiry based RSS value when inquiring device and mobile client are at distance of $1 \mathrm{~m}[6]$.

\section{POSITION ESTIMATION 3.1 Fingerprinting}

This technique is used to find the location of the device by measuring the signal strength from a set of reference points(access points).It is divided into 2 phases-offline phase and online phase. During offline phase, reference points are deployed in the workspace and Radio Map(RM) is built which is a table that contains the mapping between received signal strengths and the known positions. In online phase, the position is estimated by matching the RSS of the mobile node with that of RSS of the closest lying reference point in the RM.

In [3], three fingerprinting based algorithms are compared. Two metrices are taken into consideration: accuracy and precision. Precision is being represented by the points that are within acceptable error and accuracy by the average distance error. The algorithms compared are Neural Networks, Support Vector Machines(SVM),K-nearest Neighbors(kNN).Out of these, $\mathrm{kNN}$ gives best results.

This kNN algorithm can be explained as:-

The RSSI are taken as inputs and there are supposed outputs. Several records are collected at each location. During the running phase, RSSI at a particular point is compared to RSSI in the database and then outputs of $\mathrm{K}$ nearest neighbors are returned for calculating the final result.
Though, fingerprinting technique is most widely used, but in [7], mapping from RSSI to distance is obtained. In this, mapping is obtained by using interpolation along with motion. This motion requires lesser training time and memory as compared to fingerprinting.

\subsection{Building Radio Maps}

Building an RM for a location takes lots of efforts and time. For obtaining an RM, one has to segment the entire workspace into reference points and has to take measurements at each reference point using sensors or Bluetooth antennas. As, the RSS for a particular location is not same, it fluctuates frequently due to signal attenuation and various other factors. So, the measurement process has to be repeated many times at each location. This process can be too tedious. So, in [8], a model is proposed to avoid massive field measurement campaign. The model is explained as below-

$$
R S S=E S S-P L L
$$

Where ESS is the emitter signal strength and is the PLL path loss i.e. output gain of the sensors. Here path loss is given by-

$$
\left.P L L=20 \cdot \log \left(\frac{4 \pi d}{\lambda}\right)+\sum_{i} P_{i} \cdot A F_{i}\right)+F A F+K
$$

where $d$ is the distance on the direct path, $\lambda$ is transmission frequency of the used wireless technology, $P_{i}$ is the number of obstacles of ith type, $A F_{i}$ is the attenuation factor of the ith type of obstacle and $F A F$ is attenuation depending on the number of crossed floors. An optional attenuation factor $K$ is added to improve the correctness of the model.

For automatically selecting number of reference points and for their optimal placement in the workspace, Multi Objective Genetic Algorithm(MOGA) is used. This reduces the offline manual efforts to build a Radio Map.

\subsection{Reduction of average distance error.}

The main work done in this area is to obtain maximum accuracy which can be achieved by reducing the average error. In [9], a new method for position estimation has been introduced in which there is dynamic adaptation of path loss parameter in propagation model. Whenever there is line of sight (LOS), $n$ (path-loss exponent) is 1.8 and for non line of sight (NLOS), $n$ is 0 . Further, it is assisted by particle filter and positioning accuracy of less than $1 \mathrm{~m}$ is obtained. It performs well even when the line of sight with respect to several anchor nodes is lost.

Authors of [10] proposed an approach to monitor variations of the RSSI due to environmental changes and introduced dynamic calibration of the Bluetooth nodes to adapt to these variations. A Fuzzy Logic Unit has been taken into use to detect when these objects are in proximity to one of the reference nodes to enhance accuracy. An average distance error of $1.23 \mathrm{~m}$ is obtained.

An indoor positioning solution via RSSI probability distributions is presented in [11], [12].A Weibull function is utilized for approximating the probability distribution of BT signal strength and hence reliability of the fingerprinting database is improved. For position estimation, Histogram Maximum Likelihood algorithm is implemented. But this work could not achieve very high accuracy giving about $10 \mathrm{~m}$ of standard deviation.

In [13], a method is proposed which combines the estimation 
information from the RSS measurements with the information from motion model. Speed detection is further assisted to correct the position estimation giving average error of $4.2 \mathrm{~m}$. There is $16 \%$ improvement as compared to point Kalman filter.

An experiment is conducted in [14] to present a relationship between received power level and distance using Standard radio propagation model. Fingerprinting and lateration technique when combined in the experiment give high accuracy in distance estimation. A Gradient filter is used to minimize average error from $5.87 \mathrm{~m}$ to $2.67 \mathrm{~m}$.

An accuracy of about $70 \mathrm{~cm}$ is obtained in [2] in which Bluetooth positioning and Pedestrian Dead Reckoning (PDR) is combined. An algorithm called Peak Detection algorithm is applied to detect number of steps taken by the user carrying mobile node and also the step size. The direction of the user node is also estimated using Arc tangent function.

But the best accuracy which is achieved is that of $0.427 \pm 0.229 \mathrm{~m}$ in [7]. Here, an accurate method is described for localization for a mobile robot using Bluetooth. The mapping from RSSI to distance is obtained. An iterative trilateration method is implemented to improve accuracy.

Table 1 shows results of the algorithms implemented in the literature described above in the form of positioning accuracy.

Table 1. Positioning Accuracies

\begin{tabular}{|c|c|c|c|}
\hline S.no & Ref. no. & Method Implemented & Accuracy \\
\hline 1. & [2] & $\begin{array}{l}\text { Pedestrian Dead } \\
\text { Reckoning(PDR) }\end{array}$ & $0.7 \mathrm{~m}$ \\
\hline $\begin{array}{l}2 . \\
3 .\end{array}$ & $\begin{array}{l}{[7]} \\
{[9]}\end{array}$ & $\begin{array}{c}\text { Iterative Trilateration } \\
\text { Propagation Model, } \\
\text { Particle }\end{array}$ & $\begin{array}{c}0.427 \pm 0.229 \mathrm{~m} \\
<1 \mathrm{~m}\end{array}$ \\
\hline & & Filter & \\
\hline 4. & [10] & Fuzzy Logic Unit & $1.23 \mathrm{~m}$ \\
\hline 5. & {$[11],[12]$} & $\begin{array}{l}\text { Histogram Maximum } \\
\text { Likelihood Algorithm }\end{array}$ & $10 \mathrm{~m}$ \\
\hline 6. & [13] & Kalman Filter, Speed & $4.2 \mathrm{~m}$ \\
\hline 7. & [14] & $\begin{array}{l}\text { Detection Method } \\
\text { Fingerprinting and } \\
\text { Lateration } \\
\text { technique, Gradient Filter }\end{array}$ & $2.67 \mathrm{~m}$ \\
\hline
\end{tabular}

\section{CONCLUSION}

In this paper, we have reviewed the development that has taken place in the field of indoor localization using Bluetooth technology. Though, the positioning accuracy achieved so far is not very high as compared to GPS or Wi-Fi, but it gives a good idea of the position of the mobile node which is being tracked. The best accuracy obtained so far is that of $0.427 \pm$ $0.229 \mathrm{~m}$. Also, during an inquiry process, the communication between the devices stops for about 10 seconds which is quite a long period. So, to overcome this, the discovery time should be decreased. And by reducing the discovery time, it will be easy to take the measurements of the moving devices too. We hope this brief review will be useful to the beginners interested in this field.

\section{REFERENCES}

[1] Fazli Subhan,Halabi B Hasbullah, "Minimizing Discovery Time in Bluetooth Networks Using Localization Techniques", IEEE 2010.

[2] Suguna P. Subramanian, Juergen Sommer, Frank-Peter
Zeh, Stephen Schmitt and Wolfgang Rosential, "PBILPDR for scalable Bluetooth Indoor Localization”, 2009 Third International Conference on Next Generation Mobile Applications, Services and Technologies.

[3] Li Zhang,Xiao Liu,Jie Song,Cathal Gurrin,Zhiliang Zhu, "A Comprehensive Study of Bluetooth Fingerprinting-based Algorithms For Localization”, 2013 $27^{\text {th }}$ International Conference on Advanced Information Networking and Applications Workshops.

[4] Patrick Utsch,Thomas Liebeg, "Monitoring Microscopic Pedestrian Mobility using Bluetooth", 2012 Eighth International Conference On Intelligent Environments.

[5] Thomas King, Hendrik Lemelson, Andreas Farber and Wolfgang Effelsberg, "BluePos: Positioning with Bluetooth", $6^{\text {th }}$ IEEE International Symposium on Intelligent Signal Processing.26-28 August, 2009 Budapest, Hungary.

[6] Fazli Subhan,Halabu Hasbullah,Azat Rozyyev,Sheikh Tahir Bakhsh, "Analysis of Bluetooth Signal Parameters for Indoor Positioning Systems", 2012 International Conference on Computer and Information Science(ICCIS).

[7] Aswin N Raghavan,Harini Ananthapadmanaban,Manimaran S Sivamurugan,Balaraman Ravindran, "Accurate Mobile Robot Localization in Indoor Environments using Bluetooth", 2010 IEEE International Conference on Robotics and Autumation Anchorage Convention District May 3-8,2010,Anchorage,Alaska,USA.

[8] Christian Esposito, Domenico Cotroneo and Massimo Ficco,"Calibrating RSS-based Indoor Positioning Systems", 2009 IEEE International Conference on Wireless and Mobile Computing, Network and Communications.

[9] Javier Rodas, Carlos J. Escurdo, "Joint Estimation of Position and Channel Propagation Model Parameters in a Bluetooth Network", IEEE,2009.

[10] Ehad Akeila, Zoran Salcic, Akshya Swain, Aaron Croft and Jeremy Stott, "Bluetooth-based Indoor Positioning with Fuzzy Based Dynamic Calibration”, IEEE 2010.

[11] Ling Pei, Ruizhi Chen,Jingin Liu,Tomi Tenhunen,Heidi Kuusniemi,Yuwei Chen, "Inquiry-based Bluetooth Indoor Positioning via RSSI Probability distributions," 2010 Second International Conference on Advance in Satellite and Space Communications.

[12] Ling Pei,Ruizhi Chen,Jingbin Liu,Tomi Tenhunen, Heidi Kuusniemi,Yuwei Chen, "An Inquiry-based Bluetooth Indoor Positioning Approach for the Finnish pavilion at Shanghai World Expo 2010”, IEEE 2010

[13] Liang Chen,Heidi Kuusniemi,Yuwei Chen,Ling Pei,Tuomo Kroger and Ruizhi Chen, "Information Filter with Speed Detection for Indoor Bluetooth Positioning", IEEE 2011.

[14] Fazli Subhan,Halabi Hasbullah,Azat Rozyyev,Sheikh Tahir Bakhsh, "Indoor Positioning in Bluetooth Networks using Fingerprinting and Lateration approach", IEEE 2011. 